\title{
Energy Metabolism Mechanism of Anticardiogenic Shock Effect Component Ginsenoside Rc of Shenfu Injection on H9c2 Myocardial Injury Cells Induced by Hypoxia/Reoxygenation
}

\author{
Yu Chen, ${ }^{1}$ Yan Li, ${ }^{1}$ Guoliang Xu, ${ }^{1,2,3}$ Guangbin Shang, ${ }^{1,2}$ Hongning Liu $\mathbb{D}^{1,2}$ \\ and Li Jiang $\mathbb{1}^{1,2,3}$ \\ ${ }^{1}$ Research Center for Differentiation and Development of TCM Basic Theory, Jiangxi University of Traditional Chinese Medicine, \\ Nanchang, China \\ ${ }^{2}$ Jiangxi Provincial Key Laboratory of TCM Etiopathogenesis, Jiangxi University of Traditional Chinese Medicine, \\ Nanchang, China \\ ${ }^{3}$ Key Laboratory of Pharmacology of Traditional Chinese Medicine in Jiangxi, Nanchang, China
}

Correspondence should be addressed to Li Jiang; jiangli1009@126.com

Received 17 June 2020; Revised 18 August 2020; Accepted 25 August 2020; Published 4 September 2020

Academic Editor: Vincenzo De Feo

Copyright (c) $2020 \mathrm{Yu}$ Chen et al. This is an open access article distributed under the Creative Commons Attribution License, which permits unrestricted use, distribution, and reproduction in any medium, provided the original work is properly cited.

\begin{abstract}
Shenfu Injection (SFI) is a common drug used to treat cardiovascular diseases and has a significant effect on cardiogenic shock. Ginsenoside Rc (G-Rc) was an anticardiogenic shock effect component of SFI screened by UHPLC-Q-TOF/MS and multivariate statistical analysis and further selected by molecular docking experiment in our previous study. However, most studies on SFI in the treatment of cardiogenic shock focus on the overall efficacy, and little is known about its effective component on energy metabolism in hypoxia/reoxygenation- (H/R-) induced myocardial injury cells. Therefore, the present study was performed to investigate the dose-effect and time-effect relationship of G-Rc in protecting hypoxic injury of H9c2 cardiomyocytes, and its mechanism on the energy metabolism-related indicators, i.e., adenosine triphosphate (ATP) content, lactate dehydrogenase (LDH) release, and creatine kinase (CK) activity of the myocardial cells, was explored. In this paper, a stable and reliable H/R model of H9c2 cardiomyocytes was established. Compared with the control group, the activity of cardiomyocytes in the H/R group was significantly reduced $(P<0.01)$. The dose-effect and time-effect studies showed that G-Rc could significantly increase cell viability at certain point compared with the $\mathrm{H} / \mathrm{R}$ group $(P<0.01)$, and the optimum intervention dose and time was $3.33 \mu \mathrm{mol} / \mathrm{L}$ for $12 \mathrm{~h}$. The results concerning energy metabolism mechanism demonstrated that G-Rc pretreatment could improve ATP content, attenuate the LDH leakage, and decrease $\mathrm{CK}$ activity and apoptosis rate of $\mathrm{H} / \mathrm{R}$ cardiomyocytes. Taken together, our findings suggest that $\mathrm{G}$-Rc pretreatment can significantly protect myocardial cells from H/R injury. In addition, G-Rc is able to improve the energy metabolism ability of the injury cardiomyocytes by direct synthesis of ATP and reducing the activity of LDH, CK, and apoptosis rate. These results indicate that G-Rc may be a promising therapeutic candidate for the treatment of cardiovascular disease caused by myocardial $H / R$ injury.
\end{abstract}

\section{Introduction}

Cardiogenic shock is the main cause of death in patients with acute myocardial infarction during the hospitalization, the mortality rate of which was approximately $50 \%$ [1]. Cardiogenic shock is a syndrome characterized by a significant reduction in cardiac output and severe acute peripheral circulatory failure due to extreme cardiac dysfunction. A series of complications caused by cardiogenic shock, such as ischemia, hypoxia, energy metabolism disorders, and important organ damage, have seriously threatened human health $[2,3]$. Myocardial energy metabolism disorder is the key link of cardiogenic shock. Currently, drug therapy to optimize energy metabolism has become the therapeutic target and hotspot for preventing and treating cardiogenic shock. Shenfu Injection (SFI), which is derived from the ancient prescription Shenfu decoction, can not only effectively reduce the degree of ischemia/reperfusion injury [4], 
but also improve myocardial energy metabolism [5], and it has significant effects on cardiogenic shock and other kinds of shock in clinic $[6,7]$. Ginsenoside, as one of the main active ingredients in SFI, plays an important role in the treatment of myocardial ischemia reperfusion injury, myocardial hypertrophy, and heart failure $[8,9]$. However, due to the complexity of Chinese medicine ingredients, efficacy, and targets, most studies on SFI have focused on its overall efficacy, and its potential mechanism on myocardial energy metabolism has not been fully elucidated. Thus, the effect and the mechanism of some anticardiogenic shock component in SFI on myocardial energy metabolism are the focus of this paper. In our previous study, 15 components were detected and screened as the pharmacodynamical material basis by UHPLC-Q-TOF/MS and multivariate statistical analysis after intravenous administration of SFI in the early and middle stages of cardiogenic shock rats. And then, these components were docked with 10 targets related to the pathogenesis and drug therapy of shock through the molecular docking experiment. Ginsenoside Rc (G-Rc) was eventually selected as the study object in this paper for it had the most target and the highest docking score. G-Rc, the main effective component of ginsenoside contained in SFI, is mainly distributed in Araliaceae plants and has the functions of antitumor, anti-inflammation, antioxidation, regulating blood lipid, protecting nervous system, prevention of diabetes, and so on [10-14]. It has been reported that G-Rc can increase the content of ATP, inhibit the formation of intracellular ROS, and significantly inhibit the oxidative damage induced by tertbutyl hydroperoxide (t-bhp) in cardiomyocytes [13]. At present, there are many studies on cardiovascular effects of total ginsenoside [15-17] and some other ginsenosides like G-Rg1 [18-20] and G-Rb1 [21-23]. However, there have been few reports on the cardiovascular effects of G-Rc and its energy metabolism mechanism on myocardial injury cells induced by hypoxia/reoxygenation $(H / R)$ is still unclear. Thus, the potential cardiovascular benefits of G-Rc are still worthy of further study, because it may develop into an agent or adjuvant of existing drugs for cardiovascular disease [24]. Herein, the purpose of this study was to investigate the mechanism on the energy metabolism of G-Rc in protecting hypoxic injury of H9c2 cardiomyocytes. At first, a stable and reliable H/R model of H9c2 cardiomyocytes was established. Next, with deslanoside as the positive drug and cell viability as the evaluation index, the dose-effect and time-effect relationship of G-Rc in protecting hypoxic injury of $\mathrm{H} 9 \mathrm{c} 2$ cardiomyocytes was studied to determine the best conditions of intervention. Finally, the effect of G-Rc on energy metabolism-related indicators, i.e., ATP content, LDH release, CK activity, and apoptosis rate of the myocardial cells, was explored. This paper will contribute to the development of new drugs related to G-Rc and provide the basis for understanding that optimizing myocardial energy metabolism is an effective strategy to improve cardiogenic shock.

\section{Materials and Methods}

2.1. Materials and Reagents. Rat embryonic cardiomyoblastderived $\mathrm{H} 9 \mathrm{c} 2$ cardiomyocytes lines were obtained from the Chinese Academy of Sciences Cell Bank (Shanghai, China).
G-Rc was purchased from Vicky Biotechnology Co., Ltd. (Sichuan, China), and the positive drug deslanoside was purchased from Efa Biotechnology Co., Ltd. (Chengdu, China). The purity of these standards was more than $98.0 \%$. DMEM and RIPA tissue/cell lysate were the products of Solarbio Science and Technology Co., Ltd. (Beijing, China). Fetal bovine serum (FBS) was the product of Tianhang Biotechnology Co., Ltd. (Zhejiang, China). PBS phosphate buffer (powder) was supplied by Dingguo Changsheng Co., Ltd. (Nanchang, China). CellTiter $96^{\circledR}$ AQueous One Solution Cell Proliferation Assay (MTS) and ATP detection kit were purchased from Promega Biological Co., Ltd. (Beijing, China). The kits for determining $\mathrm{LDH}$ and creatine kinase (CK) kit were acquired from Jiancheng Bioengineering Institute (Nanjing, China). Annexin V-FITC/PI double staining apoptosis assay kit was purchased from MB Chem Company.

\subsection{H9c2 Cardiomyocytes Culture and Hypoxia/Reoxygena-} tion Model Establishment. The H9c2 cardiomyocyte line was cultured in high glucose DMEM supplemented with 15\% (v/ v) FBS. The cells were maintained in a humidified incubator (Thermo Fisher Scientific Corporation, United States) with $5 \% \mathrm{CO}_{2}$ at $37^{\circ} \mathrm{C}$, and the cells were adherent to the wall for growth. The cells were transferred when the cell density was covered to the bottom of the culture bottle up to $80-90 \%$; after $48 \mathrm{~h}$ of culture, fresh cell medium containing 15\% FBS was replaced.

The hypoxia/reoxygenation (H/R) model was built following the published method with slight modification [25]. In the previous study, we compared the two mixed gas methods and determined the establishment method of $\mathrm{H} 9 \mathrm{c} 2$ cardiomyocyte H/R model preliminarily. Briefly, the cardiomyocytes in the logarithmic growth phase were placed in a mobile trigas incubator (Hua Yi Ning Chuang Co., Ltd., Ningbo, China) and an anaerobic gas-filled bag-anoxic closed culture box (Mitsubishi Gas Chemical Company, INC, Japan) for different periods of hypoxia $(2,4,6,8$, and $10 \mathrm{~h}$ ) and then reoxygenated for $4 \mathrm{~h}$. After that, the orthogonal design experiment was used to optimize the cell hypoxia time $(6,8$, and $10 \mathrm{~h})$ and cell culture medium conditions (the proportion of serum and the content of blood glucose). Finally, we found that the establishment of $\mathrm{H} / \mathrm{R}$ model of cardiomyocytes by mobile trigas incubator method has the advantages of short time, simple operation, and stable results, and the establishment of the model can be accelerated by replacing the culture medium with glucosefree and serum-free culture medium. Moreover, the viability of LDH of cardiomyocytes increased significantly after $6 \mathrm{~h}$ $(P<0.01)$. Therefore, in this experiment, the H9c2 cardiomyocytes were cultured in a trigas incubator for anoxic culture, with glucose-free and serum-free culture medium. The cells were cultured under hypoxia for $6 \mathrm{~h}$ and then removed to the regular incubator and cultured for $4 \mathrm{~h}$ to mimic reoxygenation, and the time started when the gas concentration in the incubator reached anoxic conditions. The anoxic conditions were $94 \% \mathrm{~N}_{2}, 5 \% \mathrm{CO}_{2}$, and $1 \% \mathrm{O}_{2}$, and the reoxygenation conditions were $95 \% \mathrm{O}_{2}$ and $5 \% \mathrm{CO}_{2}$. 


\subsection{Study of Dose-Effect and Time-Effect}

2.3.1. Experimental Protocols. The H9c2 cardiomyocytes in the logarithmic growth stage were randomly divided into different groups. In the control group, H9c2 cardiomyocytes were incubated in normal DMEM for equivalent durations under normoxic conditions. The H/R group was conducted as described in the preceding section. According to the literature and our preliminary experiments, we select the appropriate concentration and intervention time of drugs to study the dose-effect and time-effect of G-Rc. In the G-Rctreated group (H/R + G-Rc), the H9c2 cardiomyocytes were pretreated with $3.33,10,33.3,100$, and $200 \mu \mathrm{mol} / \mathrm{L} \mathrm{G}-\mathrm{Rc}$ for 12,24 , and $48 \mathrm{~h}$ prior to $\mathrm{H} / \mathrm{R}$. The positive drug deslanosidetreated $(\mathrm{H} / \mathrm{R}+$ deslanoside) group were processed similarly to the G-Rc-treated group (H/R + G-Rc), and the administration concentration was $1,3.33,10,33.3$, and $100 \mu \mathrm{mol} / \mathrm{L}$.

2.3.2. Measurement of Cell Viability. Cell viability was determined by MTS assay. After the cells in each group were cultured for the corresponding time, $20 \mu \mathrm{L}$ MTS solution was added to each well and incubated at $37^{\circ} \mathrm{C}$ for $4 \mathrm{~h}$. The operation was conducted according to the instructions. The absorbance value of each hole at $490 \mathrm{~nm}$ was determined by the microplate reader (Spectra Corporation, United States), and the cell viability in each well was presented as percentage of control cells.

\subsection{Study on the Mechanism of Energy Metabolism}

2.4.1. Protocols. According to the optimal dose and time of action of H/R-induced myocardial injury cells determined above, relevant indexes of energy metabolism were detected. In the control group, H9c2 cardiomyocytes were cultured in a cell medium containing 15\% FBS under normoxic conditions for equivalent durations. The H/R group, G-Rctreated group, and deslanoside-treated group were cultured in the glucose-free and serum-free medium for $12 \mathrm{~h}$ and then placed in a three-gas incubator for hypoxic culture. The process of $H / R$ was described above.

2.4.2. Measurement of ATP Content. After the reoxygenation, wash the cells with PBS, and add $100 \mu \mathrm{L}$ RIPA solution into each well after absorbing the residual liquid. Shake the culture plate repeatedly to make the reagent fully contact with the cells. Scrape the cells and reagents off with a cell blade and centrifuge. Then, draw the standard curve and compare the luminescence value of the sample with the standard curve to obtain the ATP content in the sample.

2.4.3. Measurement of LDH Release. Appropriate amount of cell supernatant was collected after the different treatments to measure LDH using a LDH assay kits. The absorbance values of each hole at $450 \mathrm{~nm}$ were determined by microplate reader.
2.4.4. Measurement of CK Activity. After reoxygenation, the supernatant was taken to detect CK activity. Samples were operated in accordance with the CK kit strictly, and the absorbance was read at $660 \mathrm{~nm}$ using a microplate reader. The actual concentration of CK in each sample was calculated according to the standard curve.

2.4.5. Flow Cytometric Analysis of the Apoptosis Rate with Annexin V-FITC/PI Staining. To determine the apoptosis rate, an Annexin V-FITC/PI double staining apoptosis assay kit was used to detect apoptotic cells by flow cytometry, according to the manufacturer's instructions. Briefly, cardiomyocytes were cultured for $12 \mathrm{~h}$ under different conditions. After harvesting, the cells were incubated with $5 \mu \mathrm{L}$ Annexin V-EGFP for $15 \mathrm{~min}$ and $10 \mu \mathrm{L}$ PI for $5 \mathrm{~min}$ at $4^{\circ} \mathrm{C}$ under dark conditions. Flow cytometry was then performed to analyze the apoptosis rate. The apoptosis rate was detected by flow cytometry (Beckman Coulter Company, USA) within $1 \mathrm{~h}$. The data were analyzed by using FlowJo software (Tristar, CA, USA).

2.5. Statistical Analysis. The results are expressed as the means \pm standard deviation. One-way AVONA and $t$-test were used for comparison of differences between groups, and GraphPad Prism 6.0 software was used for graph and statistical analysis. Statistical significance was set at $P<0.05$.

\section{Results}

3.1. Dose-Effect and Time-Effect Relationship of Drugs in Protecting Hypoxic Injury of $\mathrm{H} 9 \mathrm{c} 2$ Cardiomyocytes. Firstly, we determined the optimal intervention dose and time of G-Rc. As shown in Figure 1(b), compared with the control group, the activity of cardiomyocytes in the H/R group was significantly reduced $(P<0.01)$. Compared with the $\mathrm{H} / \mathrm{R}$ group, $3.33 \mu \mathrm{mol} / \mathrm{L}$ G-Rc significantly increased cell viability after $12 \mathrm{~h}$ of intervention $(P<0.01)$. In the G-Rctreated group, the myocardial cell activity decreased with the increase of concentration in the range of 3.33-100 $\mu \mathrm{mol} / \mathrm{L}$. Next, we examined the effect of $3.33 \mu \mathrm{mol} / \mathrm{L}$ G-Rc intervention for 12,24 , and $48 \mathrm{~h}$ on $\mathrm{H} / \mathrm{R}$-induced myocardial injury cells. As shown in Figure 1(c), $3.33 \mu \mathrm{mol} / \mathrm{L}$ G-Rc intervention for $12 \mathrm{~h}$ can effectively increase the cell viability of H/R-induced cells $(P<0.01)$. Therefore, $12 \mathrm{~h}-3.33 \mu \mathrm{mol} / \mathrm{L}$ $\mathrm{G}-\mathrm{Rc}$ was determined as the optimal intervention condition for improving cell viability.

Subsequently, we determined the optimal dose of deslanoside for $12 \mathrm{~h}$ intervention in the same way. The effects of deslanoside at different concentrations for $12 \mathrm{~h}$ on the viability of $\mathrm{H} / \mathrm{R}$-induced cells were shown in Figure 2 . The results showed that, compared with the control group, the activity of myocardial cells in the H/R group was significantly lower after $12 \mathrm{~h}$ of intervention $(P<0.01)$. Compared with the H/R group, the cardiomyocytes activity in the deslanoside-treated group was relatively high at $12 \mathrm{~h}$ of $1 \mu \mathrm{mol} / \mathrm{L}$. Thus, we selected $1 \mu \mathrm{mol} / \mathrm{L}$ as the optimal dose of deslanoside-treated intervention for $12 \mathrm{~h}$ in the follow-up experiment. 

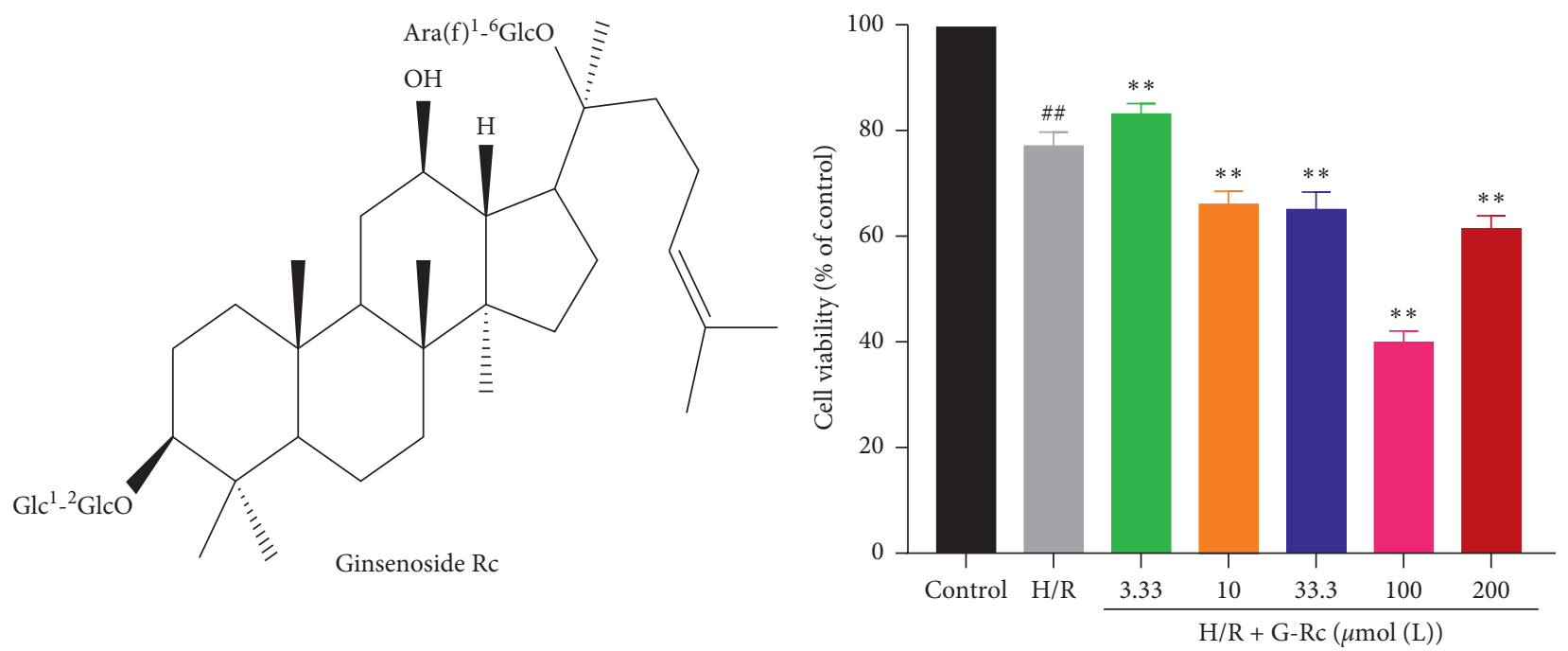

(a)

(b)

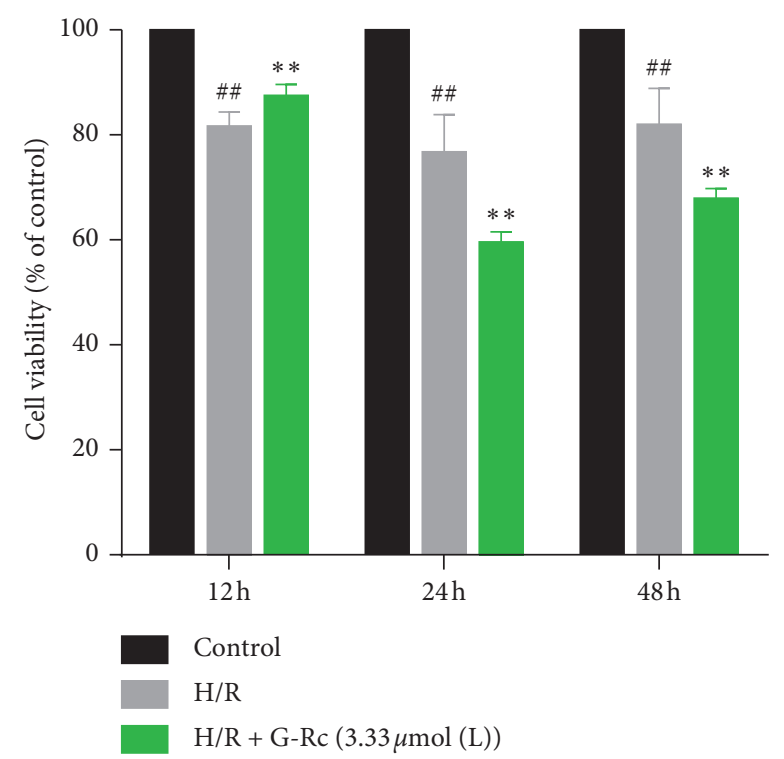

(c)

FIGURE 1: Effect of G-Rc on cell viability of H/R-induced H9c2 myocardial cell injury. (a) The chemical structure of G-Rc. (b) Cell viability of $\mathrm{H} 9 \mathrm{c} 2$ cardiomyocytes incubated with or without different concentrations of G-Rc for $12 \mathrm{~h}$ and then exposed to $6 \mathrm{~h}$ of hypoxia and $4 \mathrm{~h}$ of reoxygenation. (c) H9c2 cardiomyocytes were incubated with or without $3.33 \mu \mathrm{mol} / \mathrm{L}$ G-Rc for different incubation $(12,24$, and $48 \mathrm{~h})$ and then exposed to $6 \mathrm{~h}$ of hypoxia and $4 \mathrm{~h}$ of reoxygenation. The values are expressed as the mean $\pm \mathrm{SD}$ in six independent experiments. ${ }^{\#} P<0.05$ vs. control, ${ }^{\# \#} P<0.01$ vs. control; ${ }^{*} P<0.05$ vs. H/R group, ${ }^{* *} P<0.01$ vs. H/R group.

3.2. Effects of G-Rc on Energy Metabolism of $H / R$-Induced Myocardial Injury Cells. According to the above optimal time and concentration to intervene, we selected 12 $\mathrm{h}-3.33 \mu \mathrm{mol} / \mathrm{L} \mathrm{G}-\mathrm{Rc}$ and $12 \mathrm{~h}-1 \mu \mathrm{mol} / \mathrm{L}$ deslanoside as the basis for the determination of the following indicators.

3.2.1. G-Rc Increased the Content of ATP in H/R-Treated H9c2 Myocardial Cells. As shown in Figure 3, ATP content in the H/R group was significantly lower than that in the control group $(P<0.01)$. Compared with the $H / R$ group, ATP content in the G-Rc-treated group was significantly increased $(P<0.01)$, indicating that $\mathrm{G}-\mathrm{Rc}$ can increase the content of ATP in H/R-induced cell injury.

3.2.2. G-Rc Decreased H/R-Induced LDH Viability in H9c2 Myocardial Cells. As shown in Figure 4, LDH release in the $\mathrm{H} / \mathrm{R}$ group was significantly higher than that in the control group after $12 \mathrm{~h}$ of incubation $(P<0.01)$. Compared with the $\mathrm{H} / \mathrm{R}$ group, the viability of LDH in the G-Rc-treated group was significantly decreased $(P<0.01)$. It was speculated that G-Rc could reduce LDH release in H/R damaged cardiomyocytes and has protective effect on $\mathrm{H} 9 \mathrm{c} 2$ myocardial injury cells induced by $\mathrm{H} / \mathrm{R}$. 


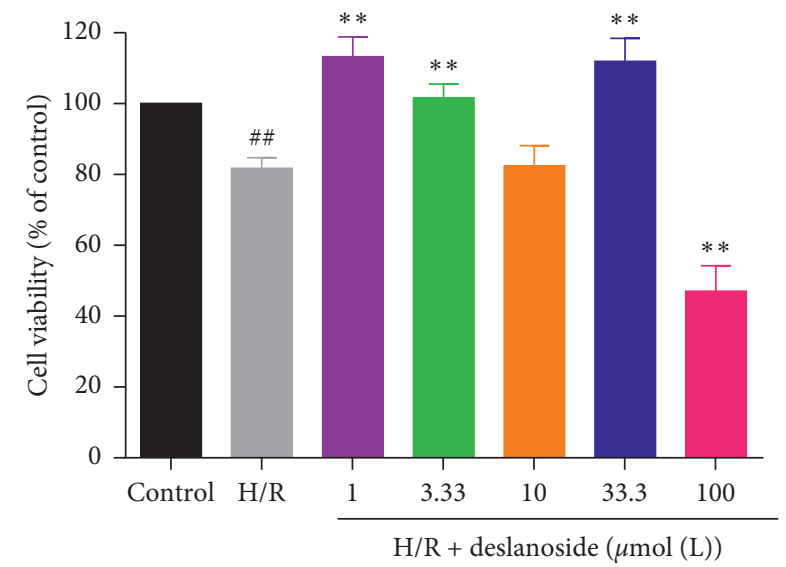

FIgURE 2: Effect of deslanoside on cell viability of H/R-induced H9c2 myocardial cell injury. H9c2 cardiomyocytes were incubated with or without deslanoside for $12 \mathrm{~h}$ and then exposed to $6 \mathrm{~h}$ of hypoxia and $4 \mathrm{~h}$ of reoxygenation. The values are expressed as the mean \pm SD in six independent experiments. ${ }^{\#} P<0.05$ vs. control, ${ }^{\# \#} P<0.01$ vs. control; ${ }^{*} P<0.05$ vs. H/R group, ${ }^{* *} P<0.01$ vs. $H / R$ group.

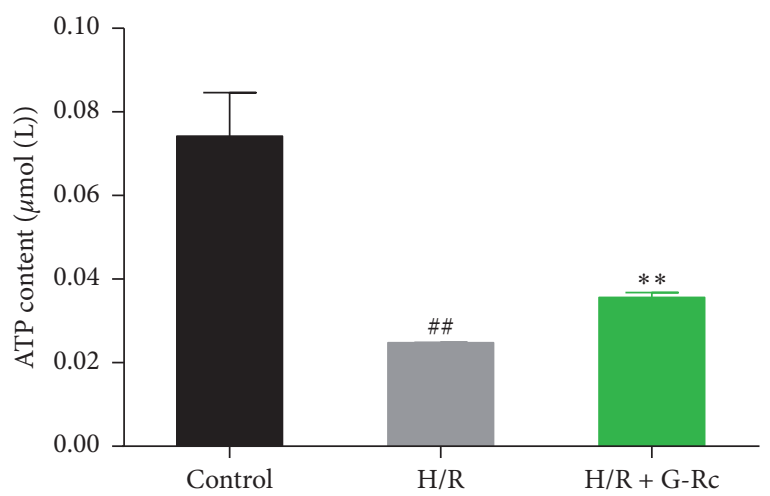

Figure 3: Effects of G-Rc on ATP content in H/R-induced cell injury. H9c2 cardiomyocytes were incubated with or without G-Rc (or the positive drug deslanoside) for $12 \mathrm{~h}$ and then exposed to $6 \mathrm{~h}$ of hypoxia and $4 \mathrm{~h}$ of reoxygenation. The values are expressed as the mean \pm SD in six independent experiments. ${ }^{\#} P<0.05$ vs. control, ${ }^{\# \#} P<0.01$ vs. control; ${ }^{*} P<0.05$ vs. H/R group, ${ }^{* *} P<0.01$ vs. $H / R$ group.

3.2.3. Effects of G-Rc on CK Activity in H/R-Induced H9c2 Myocardial Injury Cells. Figure 5 shows that CK activity in the $\mathrm{H} / \mathrm{R}$ group was higher compared with the control group after $12 \mathrm{~h}$ of incubation $(P<0.05)$. Compared with the H/R group, CK activity in the G-Rc-treated group was lower $(P<0.01)$, indicating that G-Rc could reduce the CK activity of $\mathrm{H} / \mathrm{R}$ damaged cardiomyocytes.

3.2.4. Ginsenoside Rc Reduced the Apoptosis Rate of Hypoxic Cardiomyocytes. Cellular apoptosis was detected by flow cytometry. Annexin V-FITC-/PI-cells located in the bottom left quadrant represent viable cells. In the bottom right quadrant, cellular status is located with early apoptosis (Annexin V-FITC +/PI-). As shown in Figure 6, the

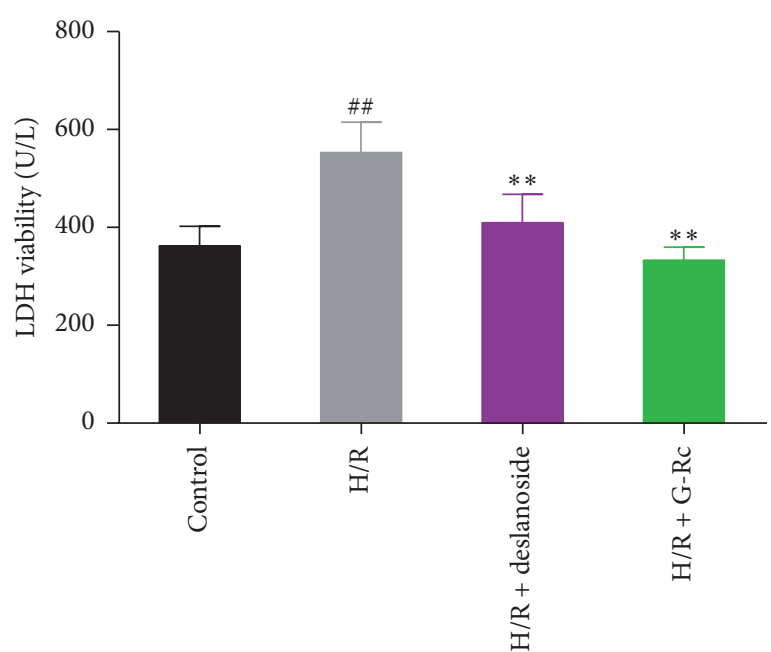

Figure 4: Effects of G-Rc on $\mathrm{LDH}$ release in $\mathrm{H} / \mathrm{R}$-induced cell injury. H9c2 cardiomyocytes were incubated with or without G-Rc (or the positive drug deslanoside) for $12 \mathrm{~h}$ and then exposed to $6 \mathrm{~h}$ of hypoxia and $4 \mathrm{~h}$ of reoxygenation. The values are expressed as the mean \pm SD in six independent experiments. ${ }^{\#} P<0.05$ vs. control, ${ }^{\# \#} P<0.01$ vs. control; ${ }^{*} P<0.05$ vs. $\mathrm{H} / \mathrm{R}$ group, ${ }^{* *} P<0.01$ vs. $\mathrm{H} / \mathrm{R}$ group.

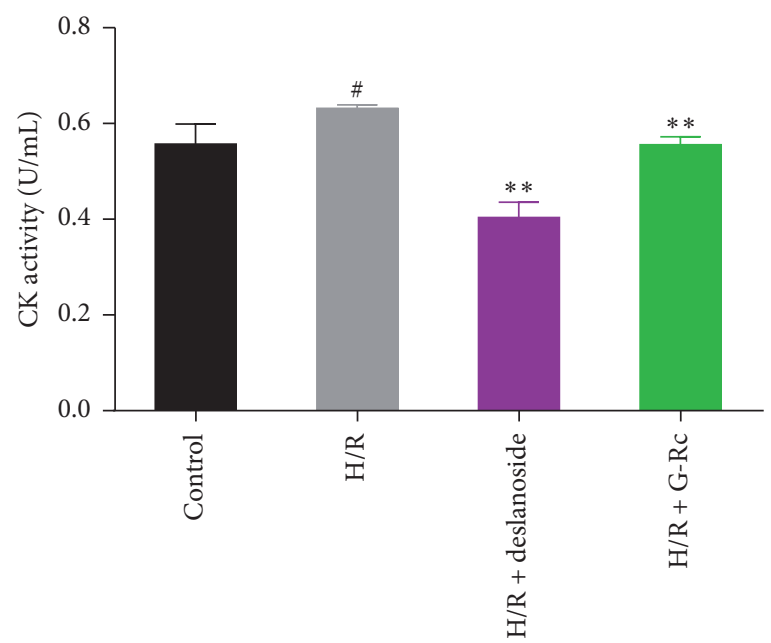

FIGURE 5: Effects of G-Rc on CK activity in H/R-induced H9c2 myocardial injury cells. H9c2 cardiomyocytes were incubated with or without G-Rc (or the positive drug deslanoside) for $12 \mathrm{~h}$ and then exposed to $6 \mathrm{~h}$ of hypoxia and $4 \mathrm{~h}$ of reoxygenation. The values are expressed as the mean $\pm \mathrm{SD}$ in six independent experiments. ${ }^{\#} P<0.05$ vs. control, ${ }^{\# \#} P<0.01$ vs. control; ${ }^{*} P<0.05$ vs. $\mathrm{H} / \mathrm{R}$ group, ${ }^{* *} P<0.01$ vs. $\mathrm{H} / \mathrm{R}$ group.

Annexin V-FITC/PI staining showed that H/R treatment significantly increased the amount of early apoptosis of $\mathrm{H} 9 \mathrm{c} 2$ cardiomyocytes compared with the control group, while $12 \mathrm{~h}$ pretreatment with $3.33 \mu \mathrm{mol} / \mathrm{L}$ G-RC effectively alleviated the H/R-induced early apoptosis.

\section{Discussion}

As a postmarketed herbal species used for more than 30 years, the composition of SFI is clear and its quality is 

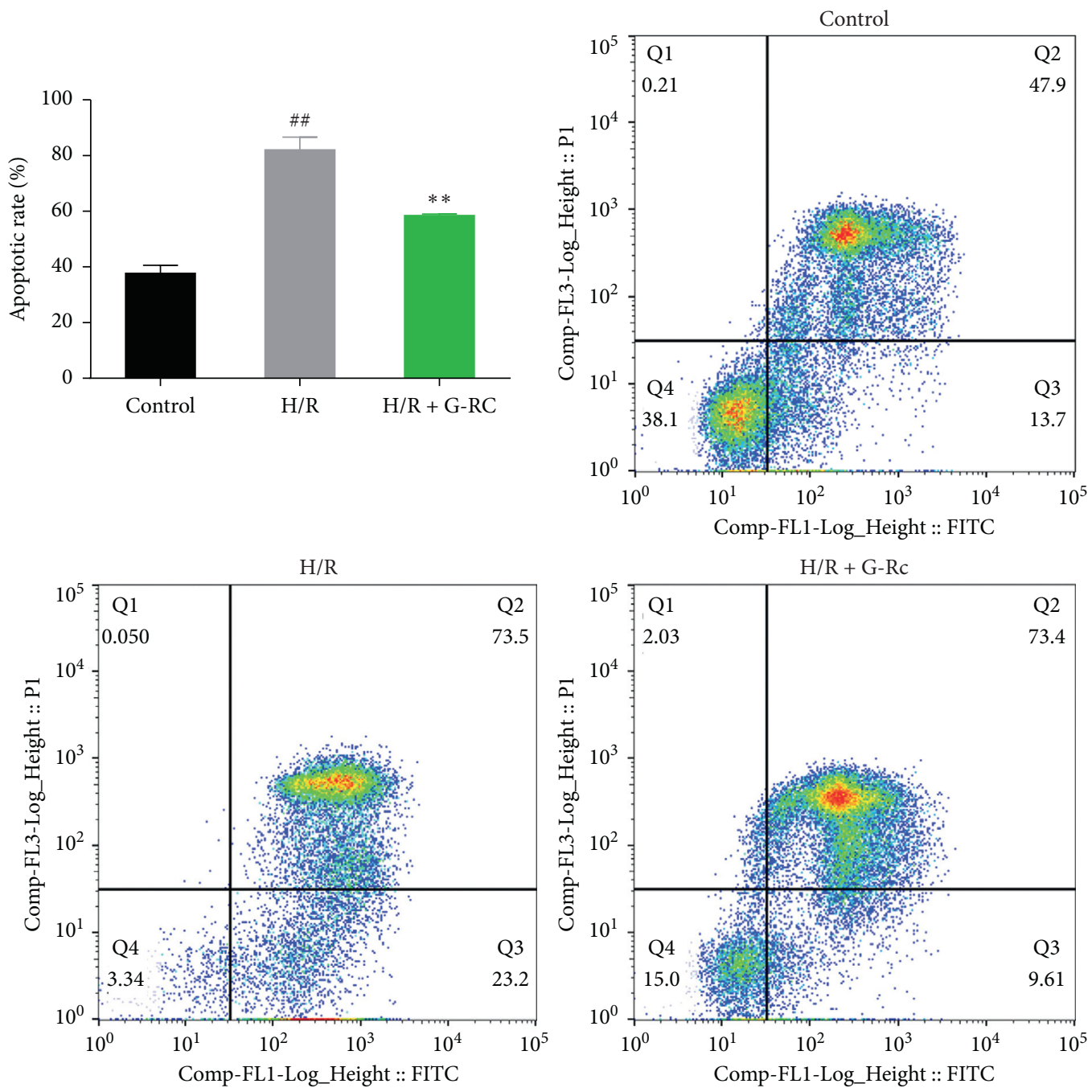

FIGURE 6: Effects of G-Rc on apoptosis rate in H/R-induced H9c2 myocardial injury cells. H9c2 cardiomyocytes were incubated with or without G-Rc for $12 \mathrm{~h}$ and then exposed to $6 \mathrm{~h}$ of hypoxia and $4 \mathrm{~h}$ of reoxygenation. The values are expressed as the mean \pm SD in six independent experiments. ${ }^{\#} P<0.05$ vs. control, ${ }^{\# \#} P<0.01$ vs. control; ${ }^{*} P<0.05$ vs. H/R group, ${ }^{* *} P<0.01$ vs. H/R group.

reliable [26]. Some research revealed that SFI has the effects of improving energy metabolism, antioxidation, and alleviating mitochondrial damage [5, 27]. Ginsenoside, one of the main active fractions in SFI, has protective effects on hypoxia-damaged cardiomyocytes, like G-Rg1, G-Rb1, and other ginsenosides [23, 28, 29]. Moreover, G-Rb1 has been proved to reduce the toxicity of aconitine to cardiomyocytes and its mechanism may be associated with cellular energy metabolism [30]. In the present study, we studied dose-effect and time-effect relationship of G-Rc in protecting hypoxic injury of $\mathrm{H} 9 \mathrm{c} 2$ cardiomyocytes for the first time and it showed that G-Rc-treated group could significantly increase cell viability compared with the H/R group. This result suggested that G-Rc pretreatment, particularly with a dose of $3.33 \mu \mathrm{mol} / \mathrm{L}$ for $12 \mathrm{~h}$, can significantly protect cardiomyocytes from $\mathrm{H} / \mathrm{R}$ injury.

To further investigate the effects of G-Rc on the H/R cardiomyocytes, we observed the energy metabolism-related indicators. Myocardial energy metabolism disorder is a key link of cardiogenic shock, and the drug therapy to optimize energy metabolism has become a therapeutic target and hotspot for prevention and treatment of cardiogenic shock. Myocardial energy metabolism refers to the process by which cardiomyocytes synthesize ATP using fatty acids and glucose and store it for further utilization. The heart is the largest energy consumer and the normal function of heart depends on the energy metabolism of the myocardium. More than $95 \%$ of the energy in heart muscle cells is produced in mitochondria and its direct energy form is ATP [31]. Decreased ATP content will aggravate the impaired myocardial energy metabolism. Our study showed that G-Rc could significantly increase the ATP content of myocardial cells after H/R injury. It suggested that G-Rc can be directly converted into ATP in cardiomyocytes, which could replenish energy for the damaged cells in a short time and enhance the metabolic capacity. Furthermore, the lack of oxygen will change the energy metabolism in the mitochondria of cardiomyocytes from aerobic oxidation to anaerobic fermentation, and a large amount of lactic acid will be produced, resulting in intracellular acidosis and the 
inability to decompose ATP. LDH is a kind of widely distributed dehydrogenase, which is a key enzyme in the synthesis of lactic acid by biological method, and mainly exists in myocardial cytoplasm and myocardial tissue [32]. $\mathrm{LDH}$ would be released when myocardial cells were damaged or necrotic, and its viability increased. After G-Rc intervention, the $\mathrm{LDH}$ viability of the damaged cardiomyocytes decreased significantly. It was speculated that the drug could inhibit the LDH viability and ensure the normal metabolism of the cells.

In addition, we determined the effect of G-Rc on CK activity in H/R cardiomyocytes. CK mainly exists in cytoplasm and mitochondria and is an important kinase directly related to energy operation, muscle contraction, and ATP regeneration in cells. Increased activity of CK can lead to energy generation disorders in cells and aggravate the generation of diseases $[33,34]$. After intervention with G-Rc, CK activity decreased, confirming that G-Rc intervention could improve cardiomyocyte energy metabolism disorder caused by H/R injury. Apoptosis by the activation of caspase was performed by I type of programmed cell death, and excessive or insufficient apoptosis can lead to cardiovascular diseases $[35,36]$. The disorder of energy metabolism can accelerate cell apoptosis. We found that G-Rc pretreatment could significantly reduce the apoptosis of cardiomyocytes in this experiment, suggesting that regulation of apoptosis of cardiomyocytes may be one of the strategies to improve energy metabolism of cardiomyocytes. Based on these results, our findings suggest that optimizing myocardial energy metabolism could be an important protective mechanism of G-Rc against cardiomyocytes from $H / R$ injury.

\section{Conclusions}

Our study indicated that G-Rc, one of SFI anticardiogenic shock components, can protect cardiomyocytes in a certain dose and time. In addition, it indicates that G-Rc can improve energy metabolism of damaged cardiomyocytes, the reason for which may by synthesizing ATP directly and reducing $\mathrm{LDH}$ release, $\mathrm{CK}$ activity, and cardiomyocytes apoptosis to enhance energy metabolism. Although more experiments are needed to elucidate the deep mechanism, as cardiomyocyte energy metabolism is related to a variety of other factors, these results suggest that G-Rc may be a promising therapeutic candidate for the treatment of cardiovascular diseases caused by myocardial H/R injury.

\section{Data Availability}

The data used to support the findings of this study are available from the corresponding author upon request.

\section{Conflicts of Interest}

The authors declare no conflicts of interest.

\section{Authors' Contributions}

Yu Chen and Yan Li contributed equally to this work.

\section{Acknowledgments}

This work was supported by grants of the National Natural Science Foundation of China (Nos. 81703823 and 81560744), Natural Science Foundation (20192BAB205110), Medical and Health Science and Technology Plan (No. 2018B131), Chinese Medicine First Class Discipline Research Fund of Jiangxi Province (JXSYLXK-ZHYAO120), and National Students' Fund for Innovation and Entrepreneurship Training Program (201910412029).

\section{References}

[1] S. V. Rao and A. Mandawat, "Percutaneous mechanical circulatory support devices in cardiogenic shock," Circulation: Cardiovascular Interventions, vol. 10, p. e4337, 2017.

[2] Y. Zhang, J. J. Fan, and M. Sun, "Analysis on the effect of shenfu injection in emergency treatment of acute myocardial infarction with cardiogenic shock," Practical Journal of Medicine \& Pharmacy, vol. 34, no. 9, pp. 795-796, 2017.

[3] J. Schumann, E. Henrich, and H. Strobl, "Inotropic agents and vasodilator strategies for the treatment of cardiogenic shock or low cardiac output syndrome," Cohrane Database of Systematic Reviews, vol. 1, 2018.

[4] Y.-Y. Wang, Y.-Y. Li, L. Li, D.-L. Yang, K. Zhou, and Y.-H. Li, "Protective effects of shenfu injection against myocardial ischemia-reperfusion injury via activation of enos in rats," Biological and Pharmaceutical Bulletin, vol. 41, no. 9, pp. 1406-1413, 2018.

[5] Y. Yu and S. R. Wang, "Effects of shenfu injection on energy metabolism of hypertrophic myocardial cells," Western Journal of Traditional Chinese Medicine, vol. 128, no. 22, pp. 3082-3076, 2013.

[6] Y.-Y. Jin, H. Gao, X.-Y. Zhang, H. Ai, X.-L. Zhu, and J. Wang, "Shenfu Injection (参附注射液) inhibits inflammation in patients with acute myocardial infarction complicated by cardiac shock," Chinese Journal of Integrative Medicine, vol. 23, no. 3, pp. 170-175, 2017.

[7] H. Cai, Z. Zhao, S. Liu et al., "Comments on Shenfu injection for improving cellular immunity and clinical outcome in patients with sepsis or septic shock," The American Journal of Emergency Medicine, vol. 37, no. 6, pp. 1207-1208, 2019.

[8] C. H. Lee and J.-H. Kim, "A review on the medicinal potentials of ginseng and ginsenosides on cardiovascular diseases," Journal of Ginseng Research, vol. 38, no. 3, pp. 161-166, 2014.

[9] L. Ma, H. Liu, and Z. Xie, "Ginsenoside Rb3 protects cardiomyocytes against ischemia-reperfusion injury via the inhibition of JNK-mediated NF- $\kappa$ B pathway: a mouse cardiomyocyte model," Plos One, vol. 9, Article ID e103628, 2014.

[10] M.-S. Lee, J.-T. Hwang, S.-H. Kim et al., "Ginsenoside Rc, an active component of Panax ginseng, stimulates glucose uptake in $\mathrm{C} 2 \mathrm{C} 12$ myotubes through an AMPK-dependent mechanism," Journal of Ethnopharmacology, vol. 127, no. 3, pp. 771-776, 2010.

[11] D. H. Kim, C. H. Park, D. Park et al., "Ginsenoside Rc modulates Akt/FoxO1 pathways and suppresses oxidative stress," Archives of Pharmacal Research, vol. 37, no. 6, pp. 813-820, 2014.

[12] J.-W. Yang and S. Kim, "Ginsenoside rc promotes anti-adipogenic activity on 3T3-L1 adipocytes by down-regulating C/ EBP $\alpha$ and PPAR $\gamma$," Molecules, vol. 20, no. 1, pp. 1293-1303, 2015. 
[13] Y. Wang, X. Liang, and Y. Chen, "Screening SIRT1 activators from medicinal plants as bioactive compounds against oxidative damage in mitochondrial function," Oxidative Medicine and Cellular Longevity, vol. 2016, Article ID 4206392, 2016.

[14] W. X. Lu, S. Q. Zhang, and S. Z. Shen, "Research progress in pharmacological effects and pharmacokinetics of ginsenoside Rc," Chinese Traditional and Herbal Drugs, vol. 43, pp. 5961-5967, 2018.

[15] J.-R. Wang, H. Zhou, X.-Q. Yi, Z.-H. Jiang, and L. Liu, “Total ginsenosides of radix ginseng modulates tricarboxylic acid cycle protein expression to enhance cardiac energy metabolism in ischemic rat heart tissues," Molecules, vol. 17, no. 11, pp. 12746-12757, 2012.

[16] Y. Sun, Y. Liu, and K. Chen, "Roles and mechanisms of ginsenoside in cardiovascular diseases: progress and perspectives," Science China Life Sciences, vol. 59, no. 3, pp. 292-298, 2016.

[17] M. Irfan, M. Kim, and M. H. Rhee, "Anti-platelet role of Korean ginseng and ginsenosides in cardiovascular diseases," Journal of Ginseng Research, vol. 44, no. 1, pp. 24-32, 2020.

[18] H.-t. Yu, J. Zhen, B. Pang, J.-N. Gu, and S.-S. Wu, "Ginsenoside Rg1 ameliorates oxidative stress and myocardial apoptosis in streptozotocin-induced diabetic rats," Journal of Zhejiang University-SCIENCE B, vol. 16, no. 5, pp. 344-354, 2015.

[19] M. Zheng, M. Zhao, L. Tang, C. Zhang, L. Song, and W. Wang, "Ginsenoside Rg1 attenuates hypoxia and hypercapnia-induced vasoconstriction in isolated rat pulmonary arterial rings by reducing the expression of $\mathrm{p} 38$," Journal of Thoracic Disease, vol. 8, no. 7, pp. 1513-1523, 2016.

[20] Z. M. Xu, C. B. Li, and Q. L. Liu, "Ginsenoside Rg1 prevents doxorubicin-induced cardiotoxicity through the inhibition of autophagy and endoplasmic reticulum stress in mice," International Journal of Molecular Sciences, vol. 19, 2018.

[21] L. Qin, J. Wang, R. Zhao, X. Zhang, and Y. Mei, "Ginsenoside$\mathrm{Rb} 1$ improved diabetic cardiomyopathy through regulating calcium signaling by alleviating protein O-GlcNAcylation," Journal of Agricultural and Food Chemistry, vol. 67, no. 51, pp. 14074-14085, 2019.

[22] S.-n. Dai, A.-j. Hou, S.-m. Zhao et al., "Ginsenoside Rb1 ameliorates autophagy of hypoxia cardiomyocytes from neonatal rats via AMP-activated protein kinase pathway," Chinese Journal of Integrative Medicine, vol. 25, no. 7, pp. 521-528, 2019.

[23] X. Zheng, S. Wang, X. Zou et al., "Ginsenoside Rb1 improves cardiac function and remodeling in heart failure," Experimental Animals, vol. 66, no. 3, pp. 217-228, 2017.

[24] M. Karmazyn, M. Moey, and X. T. Gan, "Therapeutic potential of ginseng in the management of cardiovascular disorders," Drugs, vol. 71, no. 15, pp. 1989-2008, 2011.

[25] Y. Chen, Y. Li, and W. W. Wang, "Research progress in the establishment of hypoxia and reoxygenation model of cardiomyocytes and the detection indexes related to energy metabolism," Chinese Journal of Pharmacology and Toxicology, vol. 33, p. 809, 2019.

[26] C. X. Liu, Y. Z. Hou, and X. L. Wang, "Clinical assessment of Shenfu injection loading in the treatment of patients with exacerbation of chronic heart failure due to coronary heart disease: study protocol for a randomized controlled trial," Trials, vol. 16, p. 222, 2015.

[27] Q. Zhang and C. Li, "The roles of traditional Chinese medicine: shen-fu injection on the postresuscitation care bundle,"
Evidence-Based Complementary and Alternative Medicine, vol. 2013, Article ID 319092, , 2013.

[28] Z. L. Zhang, Y. Fan, and M. L. Liu, "Ginsenoside Rg1 inhibits autophagy in H9c2 cardiomyocytes exposed to hypoxia/ reoxygenation," Molecular and Cellular Biochemistry, vol. 365, pp. 243-250, 2013.

[29] G. Dong, T. Chen, X. Ren et al., "Rg1 prevents myocardial hypoxia/reoxygenation injury by regulating mitochondrial dynamics imbalance via modulation of glutamate dehydrogenase and mitofusin 2," Mitochondrion, vol. 26, pp. 7-18, 2016.

[30] Z. Cheng, Z. Xue, and X. Bing, "Compatibility effect of aconitine and ginsenosides Rbl on energy metabolism of primary cultured myocardial cells," Modernization of Traditional Chinese Medicine and Materia Medica-World Science and Technology, vol. 17, pp. 1785-1789, 2015.

[31] X. Chen and H. W. Liu, "Reprogramming of the research process of energy metabolism in the process of myocardial cells," International Journal of Immunology, vol. 39, no. 5, pp. 484-487, 2016.

[32] H. Cao, H. Xu, G. Zhu, and S. Liu, "Isoquercetin ameliorated hypoxia/reoxygenation-induced $\mathrm{H} 9 \mathrm{C} 2$ cardiomyocyte apoptosis via a mitochondrial-dependent pathway," Biomedicine \& Pharmacotherapy, vol. 95, pp. 938-943, 2017.

[33] M. Zhao, L. Sun, X.-J. Yu et al., "Acetylcholine mediates AMPK-dependent autophagic cytoprotection in H9c2 cells during hypoxia/reoxygenation injury," Cellular Physiology and Biochemistry, vol. 32, no. 3, pp. 601-613, 2013.

[34] X. F. Liu and H. M. Ma, "Effect of UCF-101 on cardiac enzymes, oxidative stress and cardiomyocytes apoptosis induced by adriamycin in mice," Chinese Journal of Microcirculation, vol. 29, pp. 16-20, 2019.

[35] C. Thompson, "Apoptosis in the pathogenesis and treatment of disease," Science, vol. 267, no. 5203, pp. 1456-1462, 1995.

[36] T. G. Cotter, "Apoptosis and cancer: the genesis of a research field,” Nature Reviews Cancer, vol. 9, no. 7, pp. 501-507, 2009. 